\title{
Perú: Integración, especialización sectorial y sincronización de los ciclos internacionales del producto
}

\author{
Mario D. Tello
}

RESUMEN

Se analizan las interrelaciones de la integración comercial, financiera y de especialización sectorial con el grado de sincronización entre los ciclos del producto interno bruto (PIB) del Perú y los respectivos de los 31 principales países socios comerciales. El análisis se basa en la estimación de un sistema de ecuaciones simultáneas con datos de panel, en que además se considera la repercusión de los acuerdos preferenciales de comercio (APC). Los resultados muestran relaciones robustas y bidireccionales entre el grado de sincronización y el de integración financiera, entre este y la integración comercial, y de esta con el nivel de especialización sectorial. LOS APC no incidieron en las diversas variables consideradas. Estas evidencias sugieren que una mayor integración comercial en el Perú agudizaría los efectos de los ciclos del PIB de los países socios en el producto peruano en el contexto de las crisis de 2008 y 2010.

PALABRAS CLAVE

CLASIFICACIÓN JEL
Integración económica, política comercial, política financiera, especialización de la producción, convenios preferenciales, crecimiento económico, ciclos económicos, producto interno bruto, modelos matemáticos, Perú

F4, C33, O11

Mario D. Tello. centrum Católica y Departamento de Economía de la Pontificia Universidad Católica del Perú. mtello@pucp.edu.pe 


\section{I}

\section{Introducción}

Dos enfoques que abordan las repercusiones de las crisis y ciclos internacionales en una economía son aquellos basados en los modelos, regularidades empíricas y sincronización de los ciclos económicos internacionales (por ejemplo, Fidrmuc y Korhonen, 2009; y Backus, Kehoe y Kidland, 1992) entre países y aquellos que estiman los impactos de las crisis, la volatilidad o los choques (shocks) externos en el crecimiento o desempeño económico (por ejemplo, Edwards, 2007; Aghion y Banerjee, 2005; De Gregorio y Lee, 2003; y Loayza y Hnatkovska, 2003). En relación con estos últimos, el autor (Tello, 2009b) ha estimado la incidencia de los choques externos y los acuerdos preferenciales de comercio (APC) ${ }^{1}$ en el crecimiento del producto interno bruto (PIB) real por habitante de la economía peruana en el período 1950-2007.

En el estudio se concluye que los choques externos no alteraron el crecimiento económico de largo plazo del Perú durante dicho período, aunque sí afectaron al PIB por habitante y a su tasa de variación anual en el corto plazo. La intensidad de estos impactos y su duración dependieron del manejo de las políticas (o programas) de estabilización y de las magnitudes de los choques externos. Por otra parte, los APC que se implementaron a partir de los años setenta no han contribuido a la

$\square$ El presente trabajo fue elaborado en el marco de proyectos de Comercio y Pobreza en América Latina (COPLA), financiado por el Departamento de Desarrollo Internacional del Gobierno del Reino Unido (DFID) a través del Instituto de Desarrollo de Países Extranjeros (ODI, por sus siglas en inglés). El autor agradece la colaboración de Gabriel Rodríguez en un informe de Copla en el que se basa el presente trabajo. También agradece los apoyos de Hans Lavilla, Carmen Zeña y Jairo Flores y al árbitro anónimo de la Revista CEPAL por sus comentarios. ${ }^{1}$ Un acuerdo preferencial de comercio es definido como un conjunto de instrumentos que los países utilizan con el propósito de reducir (total o parcialmente) las restricciones al comercio de bienes, servicios y factores transados. Estos acuerdos pueden ser de cuatro tipos: i) unilaterales: cuando una economía reduce unilateralmente sus restricciones al comercio; ii) bilaterales o regionales: cuando dos o más países acuerdan reducir de forma discriminada (en contra de los países no miembros del acuerdo) y recíproca las restricciones al comercio; iii) sistema generalizado de preferencias: cuando un país otorga a un grupo de países reducciones de sus restricciones comerciales de manera discriminada y sin exigencia de reciprocidad de dichas reducciones, y iv) acuerdo multilateral: que es un acuerdo regional de amplia cobertura de países, como es el caso de los países miembros de la Organización Mundial del Comercio (OMC). generación de tasas mayores de crecimiento del producto por habitante y de la productividad. Los efectos de estos acuerdos por lo general fueron limitados por el tipo de modelos de desarrollo implementados, y por la nula reducción de las barreras comerciales no arancelarias. Estas restricciones comerciales continuaron limitando el acceso a los mercados de exportación de los principales países socios comerciales, a pesar de que las barreras arancelarias han disminuido progresivamente a consecuencia de esos acuerdos (unilaterales, bilaterales o regionales, y multilaterales).

En el presente trabajo, de carácter exploratorio, se aborda el tema de la crisis externa. Bajo el primer enfoque, se analiza la incidencia de la integración (comercial y financiera), el nivel de especialización sectorial y los APC en el grado de sincronización entre los ciclos del PIB de los principales socios comerciales del Perú con respecto al producto peruano. Así, de acuerdo con este enfoque, se evalúa el efecto en las correlaciones de los ciclos de los productos de los países socios que resultan de un mayor o menor volumen de flujo de bienes y de capital o del grado de diferenciación en la estructura sectorial entre dichos países. La principal conclusión derivada de la serie de evidencias reportadas para el período 1982-2006, es que los ciclos internacionales originados por choques internos de los principales socios comerciales han incidido en los ciclos del PIB real del Perú. En contraste y como segunda conclusión relevante de las estimaciones, los APC implementados por el Perú durante el período considerado no han influido de forma estadísticamente significativa en el grado de sincronización de los ciclos del PIB del Perú y del de sus principales socios comerciales.

El trabajo se compone de cinco secciones. En la sección II se resumen los aspectos teóricos que sustentan la interrelación del grado de sincronización de los ciclos del PIB y de la integración comercial y financiera con el grado de especialización sectorial entre los países que comercian. En la sección III se expone la especificación a ser estimada y se enumeran las variables a considerar y las fuentes de información. En la sección IV se resumen los resultados a manera de hipótesis dado el carácter exploratorio del trabajo. En la sección V se entregan las conclusiones. Al final de trabajo se enumeran las referencias. 


\section{II}

\section{Sincronización de los ciclos y su relación con el grado de integración comercial, financiera y de especialización sectorial: aspectos conceptuales}

Intuitivamente, a mayor grado de integración entre países, mayor será el grado de transmisión de los ciclos económicos entre ellos. El grado de integración puede ocurrir a través del intercambio de bienes y servicios o del flujo de capitales y activos financieros. Sin embargo, este argumento intuitivo no es teóricamente correcto. Más aún, existe una discrepancia notable entre las evidencias empíricas del grado de "sincronización" o "co-movimientos" del producto ${ }^{2}$ entre países y los modelos teóricos con que se trata de explicar dicho grado o movimientos.

Calderón, Chong y Stein (2007), basados en Stockman (1988), resumen parte de los argumentos teóricos ${ }^{3}$ con los componentes del coeficiente de correlación de los PIB reales de dos países, $Y i$ y $Y j$.

$$
\begin{gathered}
\rho_{i j}=\sigma_{k} \cdot\left(\sigma_{i} \cdot \sigma_{j}\right)^{-1 / 2} \cdot \sum_{k=1}^{N_{s}} w_{k i} w_{k j}+ \\
\sigma_{i j} \cdot\left(\sigma_{i} \cdot \sigma_{j}\right)^{-1 / 2} \\
w_{k i}=Y_{k i} / Y_{i} ; w_{k j}=Y_{k j} / Y_{j}
\end{gathered}
$$

donde $\rho_{i j}$ es el coeficiente de correlación de los ciclos del PIB de los países, $i$ y $j$ y $N_{\mathrm{s}}$ es el número de sectores.

El primer sumando de (1.1) refleja la contribución al coeficiente de correlación o grado de sincronización de los ciclos entre dos países debido a la incidencia de choques específicos en la industria o sector $k$ asumidos iguales para ambos países. Estos choques "aleatorios" son independientes respecto de los otros sectores y con relación al tiempo; $\sigma_{k}$ es la varianza de los choques iguales para todas las industrias $k ; \sigma_{i}$ y $\sigma_{j}$ son las varianzas de los

\footnotetext{
${ }^{2}$ Este grado es medido por el coeficiente de correlación de Pearson de los PIB de dos países o grupo de países.

${ }^{3}$ Otros resúmenes de los modelos teóricos sobre la relación de la sincronización de los ciclos internacionales y la integración comercial y financiera se encuentran en Ambler, Cardia y Zimmermann (2002); Canova y Dellas (1993); Baxter (1995); Kollman (2001); Kose y Yi (2002 y 2001).
}

PIB de cada país y $w_{k i}$ y $w_{k j}$ es la participación del sector $k$ del PIB total de cada país. El segundo sumando refleja la contribución al grado de sincronización de los ciclos de dos países debido a los choques agregados y específicos de cada país. $\sigma_{i j}$ es la covarianza de estos choques entre los dos países y $\sigma_{i j} \cdot\left(\sigma_{i} \cdot \sigma_{j}\right)^{-1 / 2}$ es la correlación debido a los choques de los dos países.

La teoría estándar de ventaja comparativa predice que cuanto mayor es el grado de integración de los países que poseen esta ventaja, mayor es el grado de especialización y el comercio se basa fundamentalmente en el comercio intersectorial (esto es, intercambio de bienes y servicios de diferentes industrias). De este modo, se espera que cambios en $w_{k i}$ estén negativamente correlacionados con cambios de $w_{k j}$ y, por lo tanto, sea negativa la contribución al grado de sincronización del primer componente debido a choques específicos de las industrias. Esto implica que a mayor integración debido al comercio interindustrial, menor será el grado de sincronización de los ciclos del PIB de las dos economías.

Contrariamente, si el comercio es dominado por ventajas competitivas y, en consecuencia, dominado por el comercio intraindustrial, entonces una mayor integración implica un mayor grado de asociación entre las participaciones de los sectores y, por consiguiente, un mayor grado de sincronización de los ciclos de los PIB de los dos países ${ }^{4}$. La correlación de los choques entre países reforzaría en este caso el efecto de los choques específicos sectoriales. Estos signos opuestos entre el grado de sincronización y el respectivo de integración dependen de si este último induce a un mayor o menor grado de especialización intersectorial ${ }^{5}$.

En la medida en que la integración financiera incida también en la especialización sectorial, esta también

\footnotetext{
${ }^{4}$ Krugman (1993) y Kose y Yi (2001), entre muchos otros desarrollan esta línea teórica.

${ }^{5}$ Cabe mencionar que la especialización en sectores es consistente con ambos tipos de comercio, intra e interindustrial. En el primer caso, implica un mayor grado de fineza en la especialización, por ejemplo, la especialización vertical (Kose y Yi, 2001) dentro de la misma industria.
} 
afectará al grado de sincronización. Así, Kalemli-Ozcan, Sørensen y Yosha (2001 y 2003) arguyen que si bien la especialización industrial provee una serie de beneficios para la economía, si la producción no está asegurada contra los riesgos de dicha especialización, la varianza del PIB que resultase de ella implicaría pérdidas del bienestar que podrían ser mayores que sus beneficios. Aunque los seguros específicos (por ejemplo, contra desastres, con contratos de precios a futuro, entre otros) pueden ser una forma de contrarrestar estos riesgos, es a través de la diversificación geográfica de las fuentes de ingresos por la vía del mercado de capitales internacionales que los países y regiones se aseguran contra el riesgo de la especialización. En consecuencia, si los capitales entre regiones e internacionales están bien integrados, los países y regiones pueden asegurarse contra los choques de las industrias/sectores permitiendo una mejor y mayor explotación de las ventajas comparativas o competitivas que poseen dichos países y regiones.

$\mathrm{Al}$ igual que en el caso de la integración comercial (en bienes y servicios), a mayor grado de integración financiera menor sería el grado de sincronización si el comercio entre países es interindustrial y mayor cuando el comercio es intraindustrial. En el primer caso se dice que la sincronización de los ciclos entre países es "asimétrica" y, en el segundo, que la sincronización es "simétrica".

El segundo componente que contribuye al grado de sincronización es el originado por los choques específicos de los países (no de los sectores o industrias). También el grado de integración comercial y financiera puede influir en este componente. Así, choques de demanda en un país, a través de sus efectos (difusores o secundarios) "externos" (spillovers), pueden incrementar la covarianza de los PIB de los países. Estos aumentos de la demanda del país $i$ implican una mayor demanda de bienes del país $j$; cuanto más alto es el grado de integración mayor será dicho efecto de demanda transmitido a los países. En consecuencia, este segundo componente - cuando $\sigma_{i j}>0$ - puede atenuar e incluso sobrepasar los efectos del primer componente si la fuente de las ventajas es la comparativa y reforzarlos si la fuente de las ventajas es la competitiva. En esos casos, incrementos de la integración comercial conducirían a aumentos en el grado de sincronización de los ciclos del PIB de las dos economías.

Frankel y Rose (1998) agregan que la coordinación de políticas entre regiones agudiza los efectos de la integración en el grado de sincronización de los ciclos internacionales. Por otra parte, Coe y Helpman (1995) y Lichtenberg y Van Pottelsberghe (1998) enfatizan la transmisión de los choques de productividad entre países. La transmisión de estos choques a través de la difusión tecnológica, los flujos de inversión extranjera y las fuentes de tecnología, se agudiza con el grado de integración comercial y financiera.

Un segundo grupo de argumentos teórico-empíricos de las interrelaciones de los grados de integración, especialización sectorial y sincronización proviene de Backus, Kehoe y Kidland (1993), quienes descubren lo que se denomina la "incógnita de cantidad". En un mundo libre de distorsiones de mercados con libre movilidad de factores y de acceso al intercambio de bienes, choques positivos que incrementen la rentabilidad de un país o de un sector de este implican movimientos de factores y de activos que inducen a correlaciones negativas entre productos. Kose y Yi (2006, 2002 y 2001) muestran que los modelos de ciclos internacionales no pueden replicar las evidencias empíricas del grado de sincronización de los países. Estos modelos predicen que a mayor integración comercial o financiera menor sería el grado de sincronización de los productos entre países.

A consecuencia de esta discrepancia entre evidencia empírica y modelos teóricos, parte de la literatura se ha concentrado en introducir "factores o condiciones" en la producción o en los mercados, de manera tal que puedan explicar el grado de sincronización entre los países. Un canal directo, antes mencionado, es el grado de especialización sectorial. De esta forma, a mayor discrepancia en la estructura de sectores productivos de los países menor sería el grado de sincronización de los ciclos de los productos. Dos canales indirectos, también descritos anteriormente, tienen lugar a través de los efectos de la integración comercial y financiera en el grado de especialización sectorial.

Los canales directos de la integración comercial y financiera provienen de los modelos estándar del comercio internacional, e introducen algunos cambios de los factores o condiciones en la producción y en los mercados. Así, por ejemplo, Kose y Yi (2001) introducen comercio de bienes dentro de una misma industria o sector, provenientes de "etapas del proceso productivo", y obtienen un mayor grado de sincronización cuando la "intensidad del comercio" se incrementa entre países. Por otra parte, Heathcote y Perri (2002a y 2002b); Calvo y Mendoza (2000), y Mendoza (2002) muestran que distorsiones en el mercado de capitales internacionales - tales como limitaciones en la habilidad de prestar o pedir prestados capitales a nivel internacional, o restricciones de liquidez que enfrentan los inversores) pueden acrecentar el grado de sincronización de los productos entre países. 
La literatura empírica acerca de la incidencia de la integración comercial y financiera, y el grado de especialización en el grado de sincronización de productos entre países es relativamente abundante. Entre los trabajos más recientes destaca en primer lugar el de Calderón, Chong y Stein (2007). En este se utiliza una muestra de 147 países en el período 1960-1999 y, mediante técnicas econométricas de corte transversal y de datos de panel, se concluye que: i) aumentos en el grado de integración o en la intensidad del comercio de bienes inducen a una mayor sincronización de los ciclos del producto entre los países; ii) la repercusión de la intensidad del comercio es mayor para la muestra de países desarrollados que para la de los países en desarrollo, y iii) la repercusión de la intensidad del comercio es más pronunciada para aquellos países con estructuras de sectores similares.

En segundo lugar, sobre la base de datos trimestrales del período 1980-1999 respecto de 24 países (seis en desarrollo, incluidos el Perú y 18 desarrollados) y de un sistema de cuatro ecuaciones simultáneas, Imbs (2004) encuentra los siguientes resultados: i) los patrones de especialización tienen importantes efectos en los ciclos económicos entre países, que son independientes del grado de integración comercial y financiera; ii) bajo una variedad de medidas de integración financiera, regiones altamente integradas financieramente presentan una mayor sincronización de los ciclos de sus productos, a pesar de su especialización sectorial, y iii) si el comercio es intraindustrial, también ocurren incrementos del grado de sincronización de los productos entre países.

Por último, García-Herrero y Ruiz (2008) —sobre la base de una muestra de 109 países ( 88 de ellos en desarrollo) en el período 1990-2004 y de un sistema de cuatro ecuaciones simultáneas- estiman la incidencia de la integración comercial, financiera y de especialización en el nivel de sincronización de los ciclos del PIB de España con aquellos de sus socios comerciales. Estos autores encuentran que: i) la intensidad del comercio y la similitud de la estructura sectorial entre España y sus países socios en comercio afectan positivamente al grado de sincronización de los ciclos de los productos entre España y sus socios comerciales, y ii) no obstante, la integración financiera afecta negativamente al nivel de sincronización. Este resultado es consistente con los modelos estándar de los ciclos internacionales.

En resumen, los aspectos teóricos y las evidencias empíricas descritos en esta sección señalan que la sincronización de los ciclos del producto de una economía con aquellos de los países con quienes esa economía intercambia bienes, servicios y activos financieros, si bien se asocia al grado de integración comercial, financiera y de especialización de la estructura sectorial entre los países que comercian, estas relaciones no son únicas, tienen componentes directos e indirectos y dependen de otros factores, tales como las fuentes que determinan el comercio, las distorsiones en los mercados de bienes, servicios y financieros, y la existencia de diversas etapas del proceso productivo.

El propósito del presente trabajo consiste en identificar empíricamente estas asociaciones e interrelaciones para el caso de la economía peruana en el período 19822006. Para ello se tomará la especificación de un sistema de ecuaciones simultáneas similar al de los dos trabajos anteriores (Imbs, 2004, y García-Herrero y Ruiz, 2008) y se agregarán los efectos de los APC. Estos acuerdos contribuyen al grado de integración comercial entre los países miembros del APC y se espera que sus efectos sean los mismos en la sincronización de los productos y en el grado de integración comercial. 


\section{III}

\section{Especificación del sistema de ecuaciones y fuentes de información}

\section{El modelo: especificación y variables básicas}

Las consideraciones teóricas — descritas en la sección anterior- han sido formalizadas y resumidas por Imbs (2004), y García-Herrero y Ruiz (2008) mediante el siguiente sistema de ecuaciones:

$$
\begin{gathered}
\rho_{j t}=\alpha_{0}+\alpha_{1} \cdot T_{j t}+\alpha_{2} \cdot S_{j t}+ \\
\alpha_{2} F_{j t}+X_{1} \cdot \phi_{1}+\varepsilon_{1 j t} ; \\
T_{j t}=\beta_{0}+\beta_{1} \cdot S_{j t}+\beta_{2} \cdot F_{j t}+ \\
X_{2} \cdot \phi_{2}+\varepsilon_{2 j t} ; \\
F_{j t}=\delta_{0}+\delta_{1} \cdot \rho_{j t}+\delta_{2} \cdot T_{j t}+X_{3} \cdot \phi_{3}+\varepsilon_{3 j t} ; \\
S_{j t}=\gamma_{0}+\gamma_{1} \cdot T_{j t}+\gamma_{2} \cdot F_{j t}+X_{4} \cdot \phi_{4}+\varepsilon_{4 j t} ; \\
\mathrm{j}=1,31 ; \mathrm{t}=1982-2006
\end{gathered}
$$

donde $\rho_{j t}, T_{j t}, S_{j t}, y F_{j t}$, denominadas "variables básicas", son el grado de sincronización de productos (medido por el coeficiente de correlación de los ciclos de los productos), la integración comercial de bienes; la integración financiera, y el grado de especialización sectorial, respectivamente, entre el Perú y su socio comercial, país 'j' en el año t; la matriz $X_{i}$ está conformada por las variables de control para cada ecuación ' $i$ ', y $\varepsilon_{i j t}$ es el error de la ecuación ' $i$ ', país 'j', año 't'. Este sistema recoge los efectos directos e indirectos y las interdependencias teóricas existentes entre el grado de sincronización de los ciclos de los productos, los grados de integración financiera y comercial y los de especialización sectorial.

La ecuación (2.1) captura los efectos totales de estos tres últimos factores y de las variables de control en el grado de sincronización de los productos. Las ecuaciones (2.2), (2.3) y (2.4) capturan los efectos indirectos de los grados de integración financiera, comercial y de especialización sectorial y las interdependencias existentes entre estas variables. Así, en la ecuación (2.2), si el comercio entre el Perú y el país 'j' es dominado por el interindustrial (y no existieran choques en los países), entonces un incremento en la especialización genera un mayor grado de integración de bienes y viceversa. De igual modo, en la ecuación (2.4), si el comercio es dominado por los flujos interindustriales, entonces un mayor grado de integración de bienes implica una más elevada especialización sectorial. Si el signo fuese el opuesto, entonces el comercio entre el Perú y el país 'j' sería dominado por los flujos intraindustriales.

Los grados de integración financiera y comercial pueden ser complementarios o sustitutos. Serán complementarios si flujos financieros o inversiones extranjeras se destinan a los sectores de exportación donde el Perú tiene ventajas comparativas. En este caso, los signos de los coeficientes de las variables $F_{j t}$ y $T_{j t}$ en las ecuaciones (2.2) y (2.3), respectivamente, serán positivos. Estos grados serán sustitutos si los flujos financieros y las inversiones extranjeras se destinan a los sectores que compiten con las importaciones. En tal caso, los signos de los coeficientes serán negativos. El grado de integración financiera también puede afectar al grado de especialización sectorial, como fue señalado en la sección anterior. Su efecto será positivo si la integración financiera induce a una especialización intraindustrial y negativo si la integración financiera induce a una especialización interindustrial.

Para la medición de los ciclos del PIB del Perú y de los países socios se usó el filtro de Hodrick-Prescott (1997) ${ }^{6}$. El grado de sincronización, $\rho_{j t}$, es el coeficiente de correlación de Pearson calculado desde el año 1970 hasta el año ' $t$ ' entre el ciclo estimado del PIB real del país socio 'j’ y el ciclo estimado del PIB real del Perú.

Los indicadores utilizados para el grado de integración comercial son dos:

\footnotetext{
${ }^{6}$ Con parámetro $\lambda=100$, que tiene el papel de penalizar la variabilidad del componente de tendencia de la variable PIB. También se realizaron estimaciones con otras dos medidas de los ciclos: la tasa de variación de los productos y los errores de una regresión cuadrática de los PIB del Perú y de los países socios. Los resultados obtenidos con estos indicadores no alteran las conclusiones del presente trabajo.
} 


$$
\begin{gathered}
T_{1 j t}=N_{t}^{-1} \cdot \sum_{i=1970}^{t}\left(X_{j i}+M_{j i}\right) /\left(Y_{j i}+Y_{i}\right) ; \\
T_{2 j t}=\left(2 \cdot N_{t}\right)^{-1} \cdot \sum_{i=1970}^{t}\left(X_{j i}+M_{j i}\right) \cdot Y_{w i} / \\
{\left[\left(Y_{j i}\right) \cdot\left(Y_{i}\right)\right] ; \quad \mathrm{t} \leq 2006}
\end{gathered}
$$

donde $N_{t}$ es el número de años desde 1970 hasta el año 't'; $X_{j i}$ y $M_{j i}$ son los valores de las exportaciones del Perú al país 'j' y las importaciones del Perú desde el país 'j', respectivamente, en el año 'i' $(\leq \mathrm{t}) ; Y_{j i}$ es el PIB en dólares del país 'j' en el año 'i'; $Y_{i}$ es el PIB en dólares del Perú en el año 'i’; $Y_{w i}$ es el PIB en dólares del mundo en el año 'i'. El primer indicador representa el tamaño del valor de los flujos comerciales de bienes relativo a los PIB del Perú y de los países socios, y el segundo relativo al PIB del mundo.

Para los grados de integración financiera y de especialización también se utilizan dos indicadores para cada grado. Estos son:

$$
\begin{gathered}
F_{1 j t}=N_{t}^{-1} \cdot \sum_{i=1982}^{t} I_{j i} /\left(Y_{j i}+Y_{i}\right) ; \\
F_{2 j t}=N_{t}^{-1} \cdot \sum_{i=1982}^{t} I_{j i} ; \\
S_{i j t}=-N_{t}^{-1} \cdot \sum_{i=1970}^{t} \sum_{k=1}^{5} / S_{k}-S_{k j} / \\
S_{2 j t}=-\sum_{i=1970}^{t} N_{t}^{-1} \cdot / \sum_{k=1}^{5} / S_{k}-\sum_{k=1}^{5} S_{k j} /
\end{gathered}
$$

donde $I_{j i}$ es la acumulación (stock) de inversión extranjera del país 'j' en el Perú en el año 'i'; $s_{k}$ y $s_{k j}$ es la participación del PIB real del sector ' $k$ ' del PIB real para el Perú y para el país 'j’. Los sectores son: agricultura, minería, manufactura, construcción y servicios. El número $N_{t}$ para los dos indicadores de inversión se inicia desde 1982, y para el resto de variables desde 1970. Los PIB sectoriales estuvieron disponibles hasta el año $2006^{7}$.

\footnotetext{
${ }^{7}$ Indicadores de las cuatro variables básicas son reportados en el cuadro A1 del anexo.
}

Los indicadores del grado de integración financiera representan el tamaño relativo (con respecto a los PIB del Perú y de sus países socios comerciales) y absoluto de la acumulación de inversión extranjera proveniente de dichos países socios del Perú. Por otra parte, los dos indicadores de especialización sectorial representan el grado de similitud de la estructura de sectores entre el Perú y sus países socios. Una menor diferencia del grado de especialización sectorial entre el Perú y el país 'j' implica un valor $S_{j t}$ cercano a cero (0) y una mayor diferencia en dicho grado implica valores negativos distantes de cero (0) de $S_{j t}$.

\section{Los acuerdos preferenciales de comercio (APC)}

Las variables que representan los APC y que se incluyen como variables de control en la matriz $X_{i}$ son de tres tipos para los acuerdos bilaterales o regionales y de dos tipos para los acuerdos del sistema generalizado de preferencias unilaterales y multilaterales. Para los dos primeros acuerdos las variables son:

- $A_{j}=$ variable discreta que toma el valor unitario para todos los años considerados siempre y cuando el Perú y el país 'j' sean miembros del acuerdo $A$, en caso contrario toma el valor cero (0). Esta variable trata de capturar el efecto "país" sobre la variable dependiente sin tomar en cuenta el acuerdo.

- $D A_{j t}=$ variable discreta que toma el valor unitario si el Perú y el país 'j' son miembros del acuerdo $A$ durante el período ' $\mathrm{t}$ ' de implementación del acuerdo, en caso contrario toma el valor de cero (0). Esta variable trata de capturar el efecto de la "creación de comercio" del acuerdo A en la variable dependiente. Este efecto, en teoría, debería ser similar al efecto del grado de integración comercial.

- $T A_{j t}=$ variable que toma el valor de la variable tiempo (toma los números correlativos de 1 a 25 para los años comprendidos entre 1982 y 2006) en el período de implementación del acuerdo $A$ del Perú y cero (0) en el resto de los períodos. Esta variable trata de capturar el efecto permanente del acuerdo en la tendencia de la variable dependiente. Las variables para los tres últimos tipos de acuerdos son:

- $A_{t}=$ variables binarias que toman valor 1 en el período de implementación del acuerdo unilateral/ multilateral o del sistema generalizado de preferencias otorgado al Perú y cero (0) en el resto de los períodos. Esta variable trata de capturar el efecto temporal del acuerdo en el nivel de la variable dependiente. 
- $\quad T A j t=$ variable que toma el valor de la variable tiempo (toma los números correlativos de 1 a $T$ para los años comprendidos entre 1982 y 2007) en el período de implementación del acuerdo $A$ del Perú y cero (0) en el resto de los períodos. Esta variable trata de capturar el efecto permanente del acuerdo en la tendencia de la variable dependiente.

Los nombres de los acuerdos $A$ considerados son ${ }^{8}$ :

- CAN, Comunidad Andina, que se inició en 1970 y está vigente desde 1971. En 1993 el Perú se retiró temporalmente de ella para reincorporarse en 1997. De acuerdo a la notación de los acuerdos comerciales, las variables correspondientes a este acuerdo son: CAN, DCAN Y TCAN.

- CANAR, Acuerdo de alcance parcial de complementación económica (ACE) entre la CAN (excluido el Estado Plurinacional de Bolivia) y la Argentina; vigente desde 2001. Las variables correspondientes a este acuerdo son: CANAR, DCANR.

- CANBr (ACE 39), Acuerdo de alcance parcial de complementación económica entre la CAN y el Brasil en 1999. Las variables correspondientes a este acuerdo son CANBR, DCANBR, TCANBR.

- ACE 38, Acuerdo de Complementación Económica Chile-Perú, vigente desde 1998. Las variables correspondientes a este acuerdo son: $\mathrm{CHI}$, DCHI y TCHI.

- atpdea, Ley de Promoción Comercial Andina y Erradicación de la Droga, que es un sistema generalizado de preferencias otorgado por los Estados Unidos de América a los países miembros de la CAN. Estas preferencias fueron iniciadas en el ATPA (Andean Trade Preferential Act,), acuerdo comercial preferencial de los Estados de la CAN en 1992, y continuadas en forma ampliada en 2001 con la ATPDEA; en consecuencia, este acuerdo toma los valores unitarios desde 1993. Las variables correspondientes a este acuerdo son: ATPDEA y TATPDEA.

- AU, Arreglo unilateral correspondiente al período liberal de la economía peruana, período 19912007. Esta variable también recoge parte de las políticas liberales o reformas estructurales que se implementaron en dicho período. Las variables correspondientes a este arreglo son AU y TAU.

\footnotetext{
${ }^{8}$ Una lista detallada de características de los acuerdos preferenciales considerados puede encontrarse en Tello (2009a).
}

- AM, Acuerdo multilateral de la Ronda Uruguay que rige a partir de 1994. Las variables correspondientes a este acuerdo son AM y TAM.

Las variables de control adicionales para la ecuación del grado de sincronización, $X_{1}$, son:

- Dif-Infla ${ }_{j t}=$ el valor absoluto de la diferencia de inflación entre el Perú y su país socio 'j', en el período ' $t$ '. Esta variable trata de capturar el efecto de convergencia en la política económica entre los dos países en el grado de sincronización de los productos de ambos.

También se introducen variables binarias para capturar los efectos de los países miembros de regiones geográficas. Estas incluyen a América del Norte, Centroamérica, Cono Sur, Unión Europea, Asia y el resto de América. Este grupo de variables es similar a la variable $A_{j t}$. El efecto del área geográfica de la región andina es recogido por la variable CAN.

\section{Variables de control}

Las variables de control para el resto de las ecuaciones $X_{i}(i=2,3,4)$ y según sea el caso son:

- $\quad Y t=$ el PIB real (en dólares de 1990) del Perú. Esta variable trata de capturar el efecto de la demanda interna o crecimiento en las variables dependientes de las ecuaciones (2.2), (2.3) y (2.4). Ese efecto puede ser pro comercial (efecto de signo positivo) $\mathrm{o}$ anticomercial (efecto de signo negativo) ${ }^{9}$. En la ecuación (2.3) también se incluye el PIB per cápita, $Y p$, del país socio del Perú, como un factor que incentiva la inversión extranjera en la nación peruana. El signo teórico esperado de este coeficiente es positivo.

- Dif- $Y p_{j t}=$ el valor absoluto de la diferencia del PIB real per cápita entre el Perú y el país socio 'j', en el período ' $t$ '. Esta variable trata de capturar el efecto de las diferencias del grado de desarrollo en las variables dependientes de las ecuaciones (2.2), $T_{j t}$ y (2.3), $F_{j t}$.

Un signo positivo del coeficiente de Dif- $Y p_{j t}$ en la ecuación del grado de integración comercial de bienes, $T_{j t}$, significa que la fuente del comercio es la ventaja comparativa y el comercio dominante es el interindustrial. Un signo negativo del coeficiente implica que la fuente del comercio es la ventaja competitiva y el comercio

\footnotetext{
${ }^{9}$ Las estimaciones de la ecuación (2.3) también incluyen el PIB per cápita, $Y p$, que representa el nivel de desarrollo del Perú como un factor de atracción de la inversión extranjera. El signo teórico esperado de este coeficiente es positivo.
} 
dominante es el intraindustrial. Una interpretación alternativa, que produce signos contrarios, es la posibilidad de que la divergencia de los niveles de desarrollo puede ampliar la diversificación de mercados, reduciendo así el grado de integración de cada país 'j'.

En el caso de la ecuación del grado de integración financiera, $F_{j t}$, cuyos indicadores miden el tamaño relativo y absoluto de la acumulación de inversión extranjera (proveniente del país 'j'), el efecto de la variable Dif- $Y p_{j t}$ tiene dos posibles interpretaciones. En la primera, similar al caso del grado de integración comercial, la variable Dif- $Y p_{j t}$ indica la fuente de ventajas internacionales (comparativa o competitiva). Así, un signo positivo del coeficiente de esta variable implica que el tamaño del acervo de la inversión extranjera proveniente del país 'j' hacia el Perú se rige por la explotación de los recursos fuentes de las ventajas comparativas que tiene el Perú. Un signo negativo significa que la explotación se realiza en los sectores con ventaja competitiva. La segunda, como fuente de atracción para la inversión extranjera, es causada por la divergencia en el nivel de desarrollo de los países ${ }^{10}$. De este modo, cuanto mayor sea la diferencia entre el PIB per cápita del Perú con respecto a su país socio, menor será la atracción del país para el inversionista extranjero, y viceversa. La evidencia aportada en el cuadro 1 muestra el dominio del signo negativo consistente con esta segunda interpretación.

En el caso de la ecuación (2.4) del grado de similitud de la estructura sectorial, el signo teórico esperado del impacto de Dif- $Y p_{j t}$ es que sea positivo. Esto es, a menor diferencia en los niveles de desarrollo entre el Perú y su país socio, menor sería la diferencia en la estructura sectorial de los países.

$$
\begin{gathered}
\operatorname{SIMILAR}_{j t}=1-\left(Y_{j t}+Y_{t}\right)^{-2} \cdot\left(Y_{t}^{2}+Y_{j t}^{2}\right) ; \\
-\infty<\operatorname{SIMILAR}_{j t} \leq 1 / 2
\end{gathered}
$$

La variable SIMILAR $_{j t}$ representa el grado de similitud (en PIB) entre el Perú y el país socio 'j'. El signo del coeficiente de esta variable en las ecuaciones (2.2) y (2.3) es similar al del diferencial del PIB per cápita, en la medida en que representa ventajas internacionales (comparativas o competitivas). Intuitivamente cabe esperar que el signo del coeficiente en la ecuación (2.4) sea positivo. La similitud entre economías que comercian implica también similitud en el grado de especialización en sectores.

\footnotetext{
${ }^{10}$ Este argumento fue expuesto por Lucas (1990).
}

$T C R b_{j t}=$ el tipo de cambio real bilateral del país Perú con el país 'j' del período 't', donde $T C R_{j t}=$ $\left[I E_{i j t} * I P C_{j t}\right] / I P C_{i t} ; E_{i j t}$ es el tipo de cambio nominal bilateral del país Perú con respecto al país 'j', definido como el precio de la moneda del país 'j' en términos de la moneda del Perú; $I E_{i j t}$ es el índice de tipo de cambio base 2000 y $I E_{i j t}=\left(E_{i j t} / E_{i j 2000}\right) * 100 ; I P C_{i t}$ es el índice de precios al consumidor del Perú en el período 't' base 2000 y $I P C_{j t}$ es el respectivo índice para el país 'j'.

Los coeficientes de esta variable representan la combinación de los efectos precios relativos de demanda y oferta en los grados de integración comercial y financiera. El coeficiente positivo significa que el efecto precio oferta ha dominado al efecto demanda y ambos grados de integración se incrementarían ante un aumento de $T C R b_{j t}$ si las inversiones extranjeras se destinaran a los sectores de exportación. El signo negativo del coeficiente significa que el efecto precio demanda es el que domina y ambos grados disminuirían ante incrementos del TCRbjt si las inversiones extranjeras se dirigieran a los sectores de exportación.

$A R A N_{j t}=$ el arancel de la nación más favorecida (NMF), promedio simple o ponderado (en porcentaje) del país importador 'j' impuesto a los bienes de exportación del Perú en el período 't'. Esta variable representa las barreras comerciales que imponen los países socios. La teoría señala que el signo del coeficiente de esta variable para los dos grados de integración será negativo siempre y cuando los flujos de inversión extranjera se destinen a los sectores de exportación.

$D I S T_{j t}=$ la distancia entre las capitales del Perú y el país socio 'j' en kilómetros. El signo teórico es similar al de los aranceles.

Finalmente, también se introdujeron como variables de control para las ecuaciones (2.2), (2.3) y (2.4) las siguientes características de los países socios ‘j’ que comercian con o invierten en el Perú:

- $L E N G_{j}=$ variable ficticia (dummy) que toma el valor ' 1 ' si el país socio ' $\mathrm{j}$ ' tiene el mismo idioma oficial que el Perú y ' 0 ' para los demás casos. El signo teórico para el coeficiente de esta variable es positivo en cada una de las tres ecuaciones.

- $B O R D_{j}=$ variable ficticia que toma valor ' 1 ' si el país socio ' $\mathrm{j}$ ' comparte límite territorial con el Perú, y '0’ para los demás casos. El signo teórico esperado del coeficiente de esta variable para las tres ecuaciones también es positivo.

- $C O L_{j}=$ variable ficticia que toma valor ' 1 ' si el país socio 'j' ha tenido relación colonial con el Perú y '0' para los demás casos. El signo del coeficiente de esta variable es igual que en los dos casos anteriores. 
- $I S L_{j}=$ variable ficticia que toma valor ' 1 ' si el país socio 'j' es una isla, y ' 0 ' para los demás casos ${ }^{11}$. El signo teórico del coeficiente de esta variable es negativo.

- $M A R_{j}=$ variable ficticia que toma valor ' 1 ' si el país socio ' $\mathrm{j}$ ' está rodeado por tierra (prácticamente sin salida al mar), y '0' para los demás casos. El signo teórico esperado de esta variable para los grados de integración comercial y financiera es negativo.

- $A R E A_{j}=$ el tamaño del país socio ‘j’ en kilómetros cuadrados.

Las fuentes de información para las variables usadas son diversas. Los PIB reales (en dólares del año 1990) y sectoriales fueron tomados de UNCTAD (2009). Los datos de la acumulación de las inversiones extranjeras provienen de INEI (2009). Los datos de las características de los países y los tipos de cambio bilaterales fueron tomados de Tello (2009a). Los flujos de exportaciones e importaciones provienen de las Naciones Unidas (2009).

\section{Hipótesis iniciales}

En el gráfico 1 se resumen las hipótesis teóricas especificadas en el sistema de ecuaciones.

En el caso de la economía peruana, cuya estructura del sector exportador es dominada por productos primarios $^{12}$ y cuyos principales socios comerciales son

${ }^{11}$ Los países socios considerados como isla son: Australia, Nueva Zelandia, el Japón y Singapur.

${ }^{12}$ En el año 2007, el 84\% del valor total exportado estaba constituido por productos primarios y el $62 \%$ por productos mineros (Tello, 2011). países industrializados ${ }^{13}$, se espera que —en ausencia de shocks de los países- preponderen las relaciones teóricas derivadas del comercio interindustrial y de las ventajas comparativas. Específicamente, que los indicadores de los grados de especialización, integración del comercio de bienes e integración financiera afecten negativamente al grado de sincronización entre los ciclos del producto del Perú y los de sus principales socios comerciales. Esto es, que los coeficientes $\alpha_{i}$ de la ecuación (2.1) sean todos negativos. De igual modo, se espera que el grado de sincronización afecte negativamente al grado de integración financiera, es decir, que $\delta_{1}$ sea negativo y que la interrelación entre el grado de especialización y el de integración comercial sea inversa. Esto es, que los coeficientes $\beta_{1}$ y $\gamma_{1}$ sean menores de cero (0). Por otra parte, dado que las empresas extranjeras también dominan en los sectores primarios de exportación del Perú (Távara y Tello, 2010), se espera una relación de complementariedad entre el grado de integración comercial y el grado de integración financiera y que, por consiguiente, los signos de los coeficientes $\beta_{2}$ y $\delta_{2}$ de las respectivas ecuaciones (2.2) y (2.3) sean positivos. Sobre la base del mismo argumento se espera que el indicador de integración financiera afecte negativamente al indicador del grado de especialización, esto es, que $\gamma_{2}$ sea menor de cero (0).

\footnotetext{
${ }^{13}$ De los 31 principales países con que comercia el Perú, 17 son países de altos ingresos. El valor exportado a estos países representó el $60 \%$ del valor total de exportaciones del año 2007.
}

Interrelaciones teóricas entre el grado de sincronización de los ciclos del PIB y el grado de especialización, integración comercial y financiera y los APC

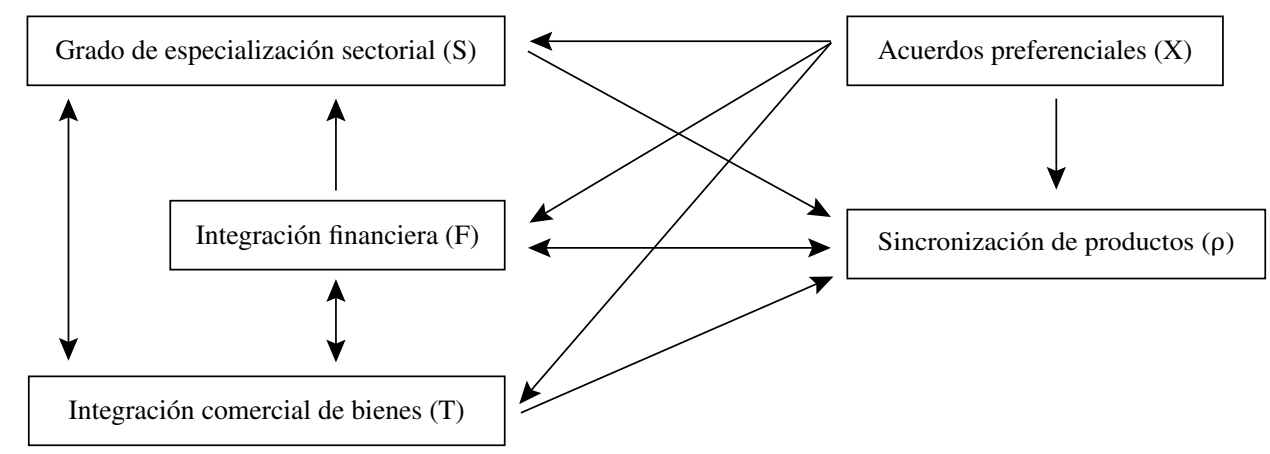

Fuente: elaboración propia.

PIB: producto interno bruto.

APC: acuerdos preferenciales de comercio. 


\section{IV}

\section{Estimaciones y resultados: el caso del Perú, 1982-2006 ${ }^{14}$}

A diferencia de los trabajos de Imbs (2004) y GarcíaHerrero y Ruiz (2008), en que se estima el sistema de ecuaciones simultáneas sin considerar las diferencias entre países, las estimaciones reportadas en el cuadro 1 presentan los coeficientes y estimadores eficientes resultantes del conjunto de regresiones realizadas con el método de mínimos cuadrados ordinarios eficientes en tres etapas (Mсо-3) $)^{15}$, utilizando los datos de panel e incorporando las diferencias entre países ${ }^{16}$ de acuerdo con la formulación de Baltagi (2005) ${ }^{17}$.

La estimación se realizó en cuatro pasos: i) estimación de los errores MCO para cada una de las cuatro ecuaciones del sistema; ii) estimación de las matrices de varianzas y covarianzas de los efectos aleatorios derivados de las diferencias entre países y los errores de cada ecuación ${ }^{18}$, usando los errores MCO; iii) determinación

\footnotetext{
${ }^{14} \mathrm{El}$ análisis empírico de esta sección es de carácter exploratorio, debido a las limitaciones de la información utilizada en el presente trabajo. Como consecuencia, las evidencias econométricas reportadas en esta sección no pueden ser interpretadas como resultados definitivos. Más bien, pueden interpretarse como hipótesis técnicas que tienen una mayor probabilidad de ser correctas, dado que las evidencias (con las imperfecciones de la información disponible) así las respaldan. Cuando se superen las limitaciones de la información se podrían verificar apropiadamente las hipótesis formuladas en esta sección.

${ }^{15}$ No se han considerado modelos de autorregresión sectorial (VARs) debido a limitaciones en el número de períodos y para fines comparativos con las estimaciones de García-Herrero y Ruiz (2008) e Imbs (2004).

16 También se realizaron estimaciones (no reportadas) con MCO, MCO de dos etapas para cada una de las ecuaciones del sistema y MCO de tres etapas para el sistema completo. Y se usaron dos medidas alternativas de los ciclos Hodrick-Prescott: los errores de las regresiones cuadráticas en el tiempo del PIB (del Perú y de sus países socios) y la tasa de variación de los respectivos PIB. Los resultados de todas estas estimaciones y medidas fueron en la mayoría de los casos similares a los aquí reportados. 17 Este método fue seleccionado para asegurar que las matrices de varianzas y covarianzas de los componentes de los errores de las ecuaciones sean definidas como positivas. Las cuatro ecuaciones fueron convertidas en un sistema de matrices $Y=Z \beta+\varepsilon$. El estimador $\beta_{e}$ y la matriz de varianzas y covarianzas, $\mathrm{V}$, de este estimador eficiente MCO-3 - de acuerdo con Baltagi (2005) - son respectivamente: $\beta_{e}=\left(Z^{*} P Z^{*}\right)^{-1}$. $\left(Z^{*} P y^{*}\right)$ y V $=\left(Z^{*} P Z^{*}\right)^{-1}$, donde $P=X^{*}\left(X^{*} X^{*}\right)^{-1} X^{*}$, y $X^{*}=\Omega^{-1 / 2} .(I \otimes X), Z^{*}=\Omega^{-1 / 2} \cdot Z ; \mathrm{y}^{*}=\Omega^{-1 / 2} . \mathrm{y} ; E\left(\varepsilon \varepsilon^{\prime}\right)=\Omega, X$ es la matriz conformada por las 40 variables predeterminadas del modelo (incluidos la constante y el tiempo).

${ }^{18}$ Donde el vector de errores $\varepsilon=\left(\varepsilon_{1 t}, \varepsilon_{2 t}, \varepsilon_{2 t} \varepsilon_{2 t}\right)$ es definido como: $\varepsilon_{j}=(I \otimes e) \mu_{j}+v_{j} ; \mu_{j}$ es el vector aleatorio de los efectos de los 31 países $(N)$ para cada ecuación ' $\mathrm{j}$ '; $v_{j}$ el vector de los errores de cada ecuación; $e$ es un vector cuya dimensión corresponde al número de años.
}

de las matrices de Cholesky de las inversas de las dos matrices anteriores ${ }^{19}$, y iv) estimación de los coeficientes del sistema de ecuaciones mediante el método de MCO-3 con variables instrumentales. Estas variables son transformaciones de las variables exógenas del sistema, las que son premultiplicadas por la matriz de Cholesky de la inversa de la matriz de varianzas y covarianzas del vector $\varepsilon$ de errores del sistema de ecuaciones ${ }^{20}$.

Se realizaron ocho regresiones por cada una de las ecuaciones del sistema resultantes de combinar los seis indicadores correspondientes a los grados de integración comercial, financiera y de especialización. En la primera columna del cuadro 1 , de cada una de las ecuaciones del sistema se muestran los coeficientes de las regresiones que tuvieron el más alto y relevante grado de ajuste. Estos coeficientes corresponden a los indicadores $\rho, T_{2}, F_{2}$, y $S_{1}$. En la segunda columna de cada ecuación se presenta el porcentaje de coeficientes positivos y estadísticamente significativos de las ocho estimaciones realizadas. En la tercera columna de cada ecuación se observa el porcentaje de coeficientes negativos y estadísticamente significativos de las ocho estimaciones efectuadas. En estas dos últimas columnas se muestra el grado de robustez estadística de los coeficientes ante variaciones de los indicadores de integración financiera, comercial y de especialización.

En las últimas dos filas del cuadro 1 se aprecian los promedios de las variables dependientes y los coeficientes de determinación de las regresiones de la primera columna.

En el gráfico 2 se muestran los resultados estadísticos de las estimaciones de los coeficientes que miden las interrelaciones entre los grados de sincronización, integración comercial y financiera y el grado de especialización sectorial del sistema de cuatro ecuaciones descritas en el cuadro 1. Estos resultados sustentan las siguientes hipótesis:

\footnotetext{
${ }^{19}$ Las matrices respectivas son: $E\left(\mu \mu^{\prime}\right)=\Sigma_{\mu}$ y $E\left(v v^{\prime}\right)=\Sigma_{v}$.

${ }^{20}$ Cabe anotar que esta matriz de Cholesky $\left(\Omega^{-1 / 2}\right)$ es función de las dos matrices de Cholesky de las matrices inversas de los errores de los efectos aleatorios de los países $\left(\Sigma^{-1 / 2}\right)$ y los errores de cada ecuación $\left(\Sigma_{v}{ }^{-1 / 2}\right)$. Específicamente: $\Omega^{-1 / 2}=\Sigma_{1}^{-1 / 2} \otimes \mathrm{P}+\Sigma_{v}{ }^{-1 / 2} \otimes \mathrm{Q}, \Sigma_{1}=\mathrm{Np} \Sigma_{u}+\Sigma_{v}$, $\mathrm{Np}=25$, es el número de años, $\mathrm{P}=\mathrm{I} \otimes \mathrm{Jp}, \mathrm{Q}=\mathrm{I}-\mathrm{P}$, Jp es una matriz cuadrada de orden $\mathrm{Np}$ cuyos elementos son iguales a $1 / \mathrm{Np}$.
} 
GRÁFICO 2

Interrelaciones empíricas para el Perú entre el grado de sincronización de los ciclos del PIB y el grado de especialización, integración comercial y financiera, y los APC
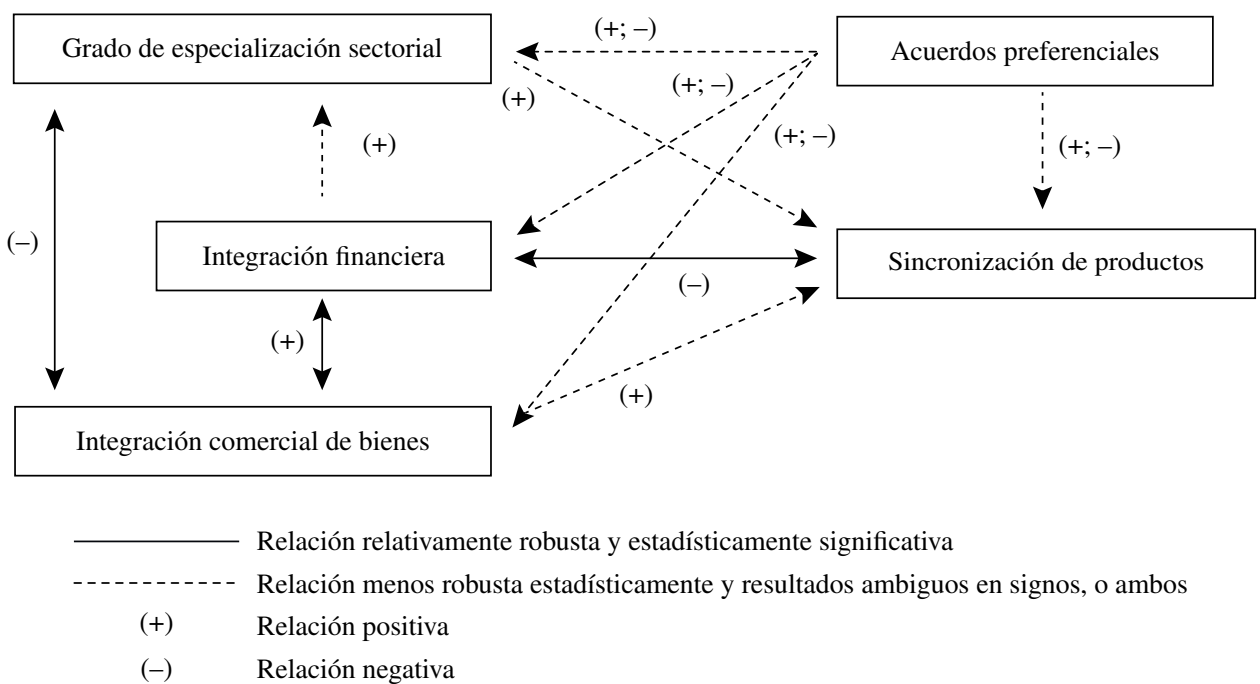

Fuente: elaboración propia.

IPC: producto interno bruto.

APC: acuerdos preferenciales de comercio.

CUADRO 1

Coeficientes estimados de las ecuaciones de los grados de sincronización, integración comercial, integración financiera y especialización.

Método eficiente Mco-3 etapas de panel, Perú, 1982-2006

\begin{tabular}{|c|c|c|c|c|c|c|c|c|c|c|c|c|}
\hline \multirow[b]{2}{*}{ Factores } & \multicolumn{3}{|c|}{$\begin{array}{l}\text { (2.1) Grado de } \\
\text { sincronización }\end{array}$} & \multicolumn{3}{|c|}{$\begin{array}{c}\text { (2.2) Grado de } \\
\text { integración comercial }\end{array}$} & \multicolumn{3}{|c|}{$\begin{array}{l}\text { (2.3) Grado de } \\
\text { integración financiera }\end{array}$} & \multicolumn{3}{|c|}{$\begin{array}{l}\text { (2.4) Grado de } \\
\text { especialización }\end{array}$} \\
\hline & Coeficiente & $\begin{array}{l}\text { Signo } \\
\text { positivo } \\
(\%)\end{array}$ & $\begin{array}{c}\text { Signo } \\
\text { negativo } \\
(\%)\end{array}$ & Coeficiente & $\begin{array}{c}\text { Signo } \\
\text { positivo } \\
(\%)\end{array}$ & $\begin{array}{l}\text { Signo } \\
\text { negativo } \\
(\%)\end{array}$ & Coeficiente & $\begin{array}{l}\text { Signo } \\
\text { positivo } \\
(\%)\end{array}$ & $\begin{array}{l}\text { Signo } \\
\text { negativo } \\
(\%)\end{array}$ & Coeficiente & $\begin{array}{l}\text { Signo } \\
\text { positivo } \\
(\%)\end{array}$ & $\begin{array}{l}\text { Signo } \\
\text { negativo } \\
(\%)\end{array}$ \\
\hline \multicolumn{13}{|c|}{ 1. Variables básicas } \\
\hline $\begin{array}{l}\text { Constante } \\
\left(\mathrm{T}_{2}\right)^{2} \mathrm{~T} \\
\left(\mathrm{~F}_{2}\right)^{2} \mathrm{~F} \\
\left(\mathrm{~S}_{1}\right)^{2} \mathrm{~S} \\
\rho\end{array}$ & $\begin{array}{c}0,1723 \\
-0,0412 \\
-0,0005^{*} \\
-0,0022\end{array}$ & $\begin{array}{l}50,0 \\
50,0 \\
25,0 \\
50,0\end{array}$ & $\begin{array}{r}12,5 \\
0,0 \\
62,5 \\
25,0\end{array}$ & $\begin{array}{l}-0,7989 \\
0,0026 \\
-0,0953 * * *\end{array}$ & $\begin{array}{l}62,5 \\
25,0\end{array}$ & $\begin{array}{r}37,5 \\
\\
0,0 \\
75,0\end{array}$ & $\begin{array}{c}120,2522 \\
70,97 * * *\end{array}$ & $\begin{array}{l}25,0 \\
75,0\end{array}$ & $\begin{array}{r}25,0 \\
0,0\end{array}$ & $\begin{array}{c}1,1847 * \\
-11,9 * * * \\
0,1099 * * *\end{array}$ & $\begin{array}{l}62,5 \\
12,5 \\
50,0\end{array}$ & $\begin{array}{r}0,0 \\
50,0 \\
25,0\end{array}$ \\
\hline \multicolumn{13}{|c|}{ 2. Acuerdos preferenciales de comercio (APC) } \\
\hline $\mathrm{CHI}$ & $-0,2664$ & 25,0 & 12,5 & $-0,8416$ & 37,5 & 12,5 & 58,5489 & 25,0 & 0,0 & $-34,5^{*}$ & 0,0 & 12,5 \\
\hline DCHI & 0,0152 & 0,0 & 12,5 & 0,0411 & 0,0 & 12,5 & 2,2246 & 0,0 & 0,0 & 0,3258 & 0,0 & 0,0 \\
\hline TCHI & $-0,1187$ & 25,0 & 0,0 & $-0,9615$ & 12,5 & 0,0 & 9,4115 & 0,0 & 0,0 & $-9,02$ & 0,0 & 0,0 \\
\hline CAN & 0,1998 & 25,0 & 37,5 & $-0,1583$ & 37,5 & 12,5 & 100,7731 & 25,0 & 0,0 & $-14,8$ & 0,0 & 0,0 \\
\hline DCAN & 0,1608 & 12,5 & 12,5 & $-1,4274 * * *$ & 12,5 & 62,5 & 131,0232 & 25,0 & 0,0 & $-24,98$ & 0,0 & 0,0 \\
\hline TCAN & $-0,0086$ & 12,5 & 12,5 & 0,0978 *** & 62,5 & 12,5 & $-11,7654$ & 0,0 & 25,0 & 1,98 & 0,0 & 25,0 \\
\hline CANBR & 0,1122 & 62,5 & 12,5 & $-1,9626^{*}$ & 12,5 & 50,0 & $-45,2430$ & 0,0 & 0,0 & $-15,11$ & 0,0 & 50,0 \\
\hline DCANBR & $-0,0505$ & 0,0 & 0,0 & $-0,1411$ & 0,0 & 0,0 & $-19,5742$ & 0,0 & 0,0 & $-2,24$ & 0,0 & 0,0 \\
\hline TCANBR & 0,0045 & 0,0 & 12,5 & 0,0078 & 0,0 & 0,0 & 0,7367 & 0,0 & 0,0 & 0,22 & 0,0 & 0,0 \\
\hline
\end{tabular}

Continúa en página siguiente 


\begin{tabular}{|c|c|c|c|c|c|c|c|c|c|c|c|c|}
\hline \multirow[b]{2}{*}{ Factores } & \multicolumn{3}{|c|}{$\begin{array}{l}\text { (2.1) Grado de } \\
\text { sincronización }\end{array}$} & \multicolumn{3}{|c|}{$\begin{array}{c}\text { (2.2) Grado de } \\
\text { integración comercial }\end{array}$} & \multicolumn{3}{|c|}{$\begin{array}{c}\text { (2.3) Grado de } \\
\text { integración financiera }\end{array}$} & \multicolumn{3}{|c|}{$\begin{array}{l}\text { (2.4) Grado de } \\
\text { especialización }\end{array}$} \\
\hline & Coeficiente & $\begin{array}{c}\text { Signo } \\
\text { positivo } \\
(\%)\end{array}$ & $\begin{array}{c}\text { Signo } \\
\text { negativo } \\
(\%)\end{array}$ & Coeficiente & $\begin{array}{l}\text { Signo } \\
\text { positivo } \\
(\%)\end{array}$ & $\begin{array}{l}\text { Signo } \\
\text { negativo } \\
(\%)\end{array}$ & Coeficiente & $\begin{array}{l}\text { Signo } \\
\text { positivo } \\
(\%)\end{array}$ & $\begin{array}{c}\text { Signo } \\
\text { negativo } \\
(\%)\end{array}$ & Coeficiente & $\begin{array}{l}\text { Signo } \\
\text { positivo } \\
(\%)\end{array}$ & $\begin{array}{c}\text { Signo } \\
\text { negativo } \\
(\%)\end{array}$ \\
\hline \multicolumn{13}{|c|}{ 2. Acuerdos preferenciales de comercio (APC) } \\
\hline $\begin{array}{l}\text { CANAR } \\
\text { DCANAR } \\
\text { TCANAR } \\
\text { ATPDEA } \\
\text { TATPDEA } \\
\text { AU } \\
\text { TAU } \\
\text { AM } \\
\text { TAM }\end{array}$ & $\begin{array}{r}-0,1790 \\
-0,2381 \\
0,0142 \\
0,0203 \\
0,0001 \\
0,0416 \\
-0,0047 \\
-0,1460 \\
0,0100\end{array}$ & $\begin{array}{r}25,0 \\
12,5 \\
12,5 \\
0,0 \\
0,0 \\
0,0 \\
12,5 \\
12,5 \\
12,5\end{array}$ & $\begin{array}{r}12,5 \\
12,5 \\
12,5 \\
0,0 \\
0,0 \\
0,0 \\
12,5 \\
12,5 \\
12,5\end{array}$ & $\begin{array}{r}0,7787 \\
0,0611 \\
-0,0127 \\
0,1829 \\
-0,0161 \\
0,2443 \\
-0,0232 \\
0,7944 \\
-0,0545\end{array}$ & $\begin{array}{r}37,5 \\
0,0 \\
12,5 \\
12,5 \\
0,0 \\
0,0 \\
0,0 \\
0,0 \\
0,0\end{array}$ & $\begin{array}{r}12,5 \\
0,0 \\
0,0 \\
0,0 \\
12,5 \\
0,0 \\
0,0 \\
0,0 \\
0,0\end{array}$ & $\begin{array}{r}-48,1836 \\
-17,2676 \\
1,4801 \\
-93,2900 \\
8,2432 \\
-71,8163 \\
6,6685 \\
-86,6109 \\
5,7680\end{array}$ & $\begin{array}{r}12,5 \\
0,0 \\
0,0 \\
0,0 \\
0,0 \\
0,0 \\
0,0 \\
0,0 \\
0,0\end{array}$ & $\begin{array}{l}0,0 \\
0,0 \\
0,0 \\
0,0 \\
0,0 \\
0,0 \\
0,0 \\
0,0 \\
0,0\end{array}$ & $\begin{array}{c}2,99 \\
5,06 \\
-0,4001 \\
17,7 \\
-1,5 \\
2,84 \\
-0,41 \\
9,11 \\
-0,58\end{array}$ & $\begin{array}{r}0,0 \\
0,0 \\
12,5 \\
0,0 \\
0,0 \\
0,0 \\
0,0 \\
0,0 \\
0,0\end{array}$ & $\begin{array}{l}0,0 \\
0,0 \\
0,0 \\
0,0 \\
0,0 \\
0,0 \\
0,0 \\
0,0 \\
0,0\end{array}$ \\
\hline \multicolumn{13}{|c|}{ 3. Variables de control } \\
\hline $\begin{array}{l}\text { Y } \\
\text { YP } \\
\text { DIF -YP } \\
\text { DIF-INFLA } \\
\text { TCRB } \\
\text { ARAN } \\
\text { TIME } \\
\text { DIST } \\
\text { LENG } \\
\text { BORDER } \\
\text { MAR } \\
\text { SIMILAR } \\
\text { AREAS } \\
\text { COL } \\
\text { ISL } \\
\text { América del Norte } \\
\text { Centroamérica } \\
\text { Cono Sur } \\
\text { Resto de América } \\
\text { Unión Europea (UE) } \\
\text { Asia }\end{array}$ & $\begin{array}{l}0,1273 \\
0,0915 \\
0,3526 * * * \\
-0,0058 \\
-0,232^{* *} \\
-0,201\end{array}$ & $\begin{array}{r}62,5 \\
37,5 \\
62,5 \\
0,0 \\
25,0 \\
37,5\end{array}$ & $\begin{array}{l}12,5 \\
37,5 \\
37,5 \\
75,0 \\
75,0 \\
37,5\end{array}$ & \begin{tabular}{|l}
$-0,1 \mathrm{E}-11$ \\
$0,33 \mathrm{E}-6^{* * *}$ \\
\\
$0,0539^{*}$ \\
0,0025 \\
$0,1057^{* * *}$ \\
$-0,0005^{* * *}$ \\
$-1,8506^{* *}$ \\
$1,5706^{*}$ \\
$1,8465^{* * *}$ \\
$0,858 \mathrm{E}-10^{* * *}$ \\
$1,396^{* * *}$ \\
0,1558 \\
0,8559
\end{tabular} & $\begin{array}{r}37,5 \\
\\
37,5 \\
\\
50,0 \\
62,5 \\
75,0 \\
37,5 \\
0,0 \\
37,5 \\
87,5 \\
37,5 \\
100,0 \\
25,0 \\
12,5\end{array}$ & $\begin{array}{r}12,5 \\
62,5 \\
\\
12,5 \\
12,5 \\
12,5 \\
50,0 \\
87,5 \\
25,0 \\
12,5 \\
62,5 \\
0,0 \\
25,0 \\
37,5\end{array}$ & $\begin{array}{c}-0,16 \mathrm{E}-10 \\
0,0354^{* * *} \\
-0,0329^{* * *} \\
\\
3,8021^{* *} \\
0,0451 \\
-4,8353^{*} \\
-0,0185 \\
-3,8455 \\
-99,6162 \\
-185,50^{* *} \\
0,248 \mathrm{E}-7^{* * *} \\
-177,33^{* * *} \\
412,55^{* * *} \\
46,62\end{array}$ & $\begin{array}{r}0,0 \\
75,0 \\
0,0 \\
\\
37,5 \\
25,0 \\
0,0 \\
50,0 \\
25,0 \\
0,0 \\
25,0 \\
87,5 \\
0,0 \\
75,0 \\
0,0\end{array}$ & $\begin{array}{r}50,0 \\
0,0 \\
75,0 \\
\\
37,5 \\
50,0 \\
37,5 \\
25,0 \\
25,0 \\
25,0 \\
50,0 \\
12,5 \\
100,0 \\
0,0 \\
25,0\end{array}$ & $\begin{array}{l}-42,9715^{*} \\
-0,0020 \\
-1,0539 \\
33,801^{* *} \\
16,3041 \\
-0,27 \mathrm{E}-8^{* * *} \\
58,492^{* * *} \\
-45,717 * * \\
3,556\end{array}$ & $\begin{array}{r}0,0 \\
0,0 \\
0,0 \\
75,0 \\
0,0 \\
0,0 \\
62,5 \\
0,0 \\
50,0\end{array}$ & $\begin{array}{r}62,5 \\
50,0 \\
37,5 \\
0,0 \\
0,0 \\
100,0 \\
0,0 \\
50,0 \\
0,0\end{array}$ \\
\hline Prom. dep. ${ }^{\mathrm{a}}$ & \multicolumn{3}{|c|}{0,064} & & 0,001 & 0,687 & & $3 \mathrm{E}-4$ & 77,190 & & $-36,834$ & $-37,107$ \\
\hline$\overline{R^{2}}$ & 0,635 & & & 0,944 & & & 0,676 & & & 0,885 & & \\
\hline
\end{tabular}

Fuente: elaboración propia.

Notas: todas las regresiones se hicieron sobre la base de 775 observaciones.

i) Con excepción de la primera ecuación donde solo hay una dependiente, en las demás ecuaciones se muestra el promedio de las dos opciones de dependientes usadas: 2,2 (T1/T2); 2,3 (F1, F2) y 2,4 (S1/S2).

ii) Los coeficientes estimados de cada ecuación corresponden a las regresiones que utilizan los indicadores: $\rho, T_{2}, F_{2}$ y $S_{1}$.

a Promedio de la variable dependiente. La nomenclatura de los acuerdos comerciales y las variables de control son descritas en la sección III de este artículo.

* nivel de significancia de $10 \%$,

***nivel de significancia de $5 \%$

*** nivel de significancia de $1 \%$.

PIB: Producto interno bruto.

CANAR: Acuerdo de alcance parcial de complementación económica entre los gobiernos de las repúblicas de Colombia, Ecuador, Perú y Venezuela, países miembros de la Comunidad Andina, y el Gobierno de la República Argentina.

CANBR: Acuerdo de alcance parcial de complementación económica entre los gobiernos de las repúblicas de Colombia, Ecuador, Perú y Venezuela, Países Miembros de la Comunidad Andina, y el Gobierno de la República Federativa del Brasil.

CHI: Acuerdo de Complementación Económica Chile-Perú.

ATPDEA: Ley de Promoción Comercial Andina y Erradicación de la Droga.

AU: Acuerdo unilateral correspondiente a los períodos liberales de la economía peruana.

AM: Acuerdo multilateral de la Ronda Uruguay. 
H1: (sobre el grado de sincronización). Cuando se toman en cuenta los efectos de los países, las repercusiones de la integración comercial y el grado de especialización derivados de la teoría de ventajas comparativas basadas en recursos naturales son dominados por los shocks de los países y producen efectos relativamente robustos estadísticamente ${ }^{21}$ en el grado de sincronización de los ciclos de los PIB del Perú y el respectivo de cada uno de los 31 países principales socios comerciales ${ }^{22}$. Así, incrementos en la integración comercial, en el grado de similitud en la estructura sectorial, o en ambos, conducen a un mayor grado de sincronización de los ciclos de los productos. Por otra parte, el efecto del grado de la integración financiera es más robusto que el de los grados anteriores dominando al parecer el efecto del comercio interindustrial. Como consecuencia, a mayor grado de integración financiera, menor es el grado de sincronización de los ciclos.

Específicamente, los porcentajes de coeficientes positivos estadísticamente significativos para $T_{i}$ y $S_{i}$ $(\mathrm{i}=1,2)$ fueron del $50 \%$ y los negativos para $F_{i}$ fueron del $62,5 \%$. Una implicancia de esta evidencia es que el grado de sincronización de los ciclos internacionales entre productos se ha incrementado con el mayor grado de integración comercial y que por lo tanto es posible que las crisis de 2008 y de 2010, esta última originada en los países desarrollados, afecten a los ciclos del PIB del Perú.

H2: (interrelaciones entre variables básicas). La evidencia muestra una variedad de interrelaciones, en su mayoría estadísticamente robustas, entre los grados de sincronización, la integración comercial y financiera y el grado de especialización sectorial. Por una parte, las interrelaciones entre la integración comercial y el grado de especialización, aquellas entre el grado de integración comercial y financiera, y las interrelaciones entre el grado de sincronización y la integración financiera son estadísticamente robustas ${ }^{23}$ y bidireccionales. Por otra, la incidencia de la integración financiera en el grado de especialización es menos robusta, predominando una relación positiva entre ambos indicadores.

\footnotetext{
${ }^{21}$ Esto es, cuando el porcentaje de coeficientes estadísticamente significativos es $50 \%$ o más.

${ }^{22}$ Nótese que la similitud en el grado de especialización sectorial entre países se acrecienta cuando $S_{i}$ se aproxima a cero $(0)$.

${ }^{23} \mathrm{Se}$ ha considerado una relación bicausal estadísticamente robusta de las variables (T, F, S) cuando la suma de los porcentajes de los (dos) coeficientes relevantes estadísticamente significativos (de T, F o S) superan el $100 \%$.
}

La interrelación entre la integración comercial y la integración financiera indica complementariedad entre el flujo de bienes y el de inversión extranjera. Esto es, la relación entre los indicadores de integración comercial y financiera para el Perú en el período 1982-2006 ha sido positiva. Por otra parte, la mayor diferencia en el grado de especialización sectorial ha producido un mayor grado de integración comercial y este a su vez ha incrementado el grado de especialización sectorial. Cabe reiterar que los indicadores $S_{j t}$ son valores negativos y cuando decrecen ello implica un mayor diferencial en el grado de especialización del Perú con respecto a su país socio comercial. La evidencia también es robusta con respecto a la incidencia del nivel de sincronización de los ciclos en el grado de integración financiera, aunque la incidencia de esta es menos robusta en el grado de especialización sectorial. Sin embargo, en este último caso la integración financiera ha inducido a una mayor similitud en la estructura de los sectores productivos. Este resultado es consistente con el mayor grado de diversificación sectorial de las inversiones extranjeras en el Perú ${ }^{24}$.

H3: (efecto de los acuerdos preferenciales de comercio). Prácticamente la mayoría de los APC no han tenido incidencia estadística robusta en el grado de sincronización de los ciclos de los productos, la integración comercial y financiera y el grado de especialización sectorial.

Solo los acuerdos de la Comunidad Andina (CAN) y el acuerdo de complementación económica entre la CAN y el Brasil han incidido de manera estadísticamente robusta en el grado de integración comercial y en los grados de sincronización y de especialización sectorial. En el segundo acuerdo fueron los efectos de los países, y no el acuerdo propiamente tal, los que afectaron a dichos grados. El efecto dominante respecto del primer acuerdo en el grado de integración fue el efecto permanente de la CAN que creó más comercio con sus países miembros.

H4: (efectos de las variables de control en el grado de sincronización). El grado de sincronización de los ciclos de los productos del Perú y de sus países socios ha sido mayor en relación con los países de América del Norte y los del Cono Sur y menor con respecto a los países de la Unión Europea y del resto de América.

\footnotetext{
${ }^{24}$ En el período 2001-2005, el 31,7\% de los flujos de inversión extranjera fueron dirigidos al sector minero, el $54,1 \%$ al sector de telecomunicaciones y un 13,8\% al sector servicios (Távara y Tello, 2010).
} 
En todas estas regiones los porcentajes de coeficientes estadísticamente significativos fueron del $62,5 \%$ o más. Además, la evidencia sustenta de forma robusta que el grado de sincronización se incrementa cuando las economías reducen el diferencial de las tasas de inflación.

H5: (efectos de las variables de control en el grado de integración comercial). Las estimaciones reportadas en el cuadro 1 señalan que las diferencias en los niveles de desarrollo entre países o el grado de similitud (en tamaño del PIB) aunados a características geográficas (como distancia, área geográfica y mar) y culturales (como el lenguaje) de los países que han comerciado con el Perú, han incidido de forma estadísticamente significativa y robusta en el grado de integración comercial entre estos países.

La evidencia deja ver que incrementos en las diferencias del nivel de desarrollo o del grado de similitud en el tamaño del PIB de los países acrecientan la diversificación de mercados y, por consiguiente, el comercio de bienes y servicios entre el Perú y su socio comercial. Por otra parte, restricciones (por ejemplo, mayores costos de transporte debidos a la mayor distancia de los países socios respecto del territorio peruano o a la imposición de aranceles) al comercio han limitado el grado de integración comercial, mientras que el tamaño del territorio de los países, el tipo de cambio real y el hecho de tener lenguajes diferentes lo han promovido. La incidencia del resto de variables de control en el grado de integración comercial no fue estadísticamente robusta.

H6: (efectos de las variables de control sobre el grado de integración financiera). De acuerdo con las predicciones de las nuevas teorías de crecimiento endógeno, aumentos en las diferencias en los niveles de desarrollo entre el Perú y sus socios comerciales no han incentivado un mayor grado de integración financiera y, en consecuencia, han afectado negativamente al tamaño relativo y absoluto de los flujos de inversión extranjera provenientes de los países socios. De la misma manera, países socios comerciales con PIB de tamaños similares al PIB del Perú o con altos niveles de PIB per cápita han incidido positivamente en la integración financiera. Excepto por las variables distancia, áreas geográficas, relaciones de coloniaje y el tamaño del PIB del Perú, la incidencia del resto de las variables de control en el grado de integración financiera no ha sido estadísticamente robusta.

Lucas (1990) provee argumentos teóricos y empíricos que sustentan la tesis de que los flujos de inversión extranjera son mayores entre países ricos, y menores entre países ricos y pobres o entre países pobres. Los resultados para el caso peruano concuerdan con estos argumentos, que señalan que disponer de recursos puede no ser suficiente para que el productor extranjero invierta en los países pobres. La dotación de capital humano y los conocimientos tecnológicos también pueden incidir en la rentabilidad de los sectores de los países, incluso en aquellos que no poseen ventajas comparativas. En ese sentido, el grado de robustez estadística ha sido más elevado para países socios con mayor grado de desarrollo que el tamaño del PIB del Perú, que no incide positivamente en la integración financiera.

Por otra parte, la evidencia también sugiere con cierta robuste $^{25}$ que la integración financiera se acrecienta con países socios distantes del Perú (por ejemplo, de las regiones de América del Norte, Europa y $\mathrm{Asia}^{26}$ ) y con países con relaciones de coloniaje, y se reduce con aumentos de los aranceles o el tamaño geográfico de los países socios.

En el caso de los efectos de las variables de control en el grado de especialización sectorial, los resultados estadísticos muestran ciertas inconsistencias con las teorías de las ventajas competitivas y los signos esperados de los coeficientes. Así, por ejemplo, la evidencia es estadísticamente robusta con respecto a la incidencia positiva de la similitud entre el país socio comercial y el Perú en términos del tamaño del PIB en la diferencia en el grado de especialización de estos países. Sin embargo, la magnitud del coeficiente del grado de similitud es muy pequeña. De manera similar, se produjeron incidencias positivas en las diferencias en la estructura sectorial, aunque con menor robustez estadística para las variables de relaciones de coloniaje y distancia geográfica del país socio con el Perú, y con el PIB del Perú. En ese último caso, la magnitud del coeficiente de la variable también es muy pequeña. Por otra parte, las variables de país socio fronterizo y la de área geográfica de los socios incidieron negativamente y con robustez estadística en la diferencia de los grados de especialización de sectores. Los resultados para el resto de las variables fueron menos robustos.

\footnotetext{
${ }^{25}$ Porcentaje de coeficientes estadísticamente significativos mayores o iguales al $50 \%$.

${ }^{26}$ Más del 50\% del valor de las exportaciones del Perú tienen como destino los mercados de los Estados Unidos de América, la Unión Europea, el Japón y China.
} 


\section{V}

\section{Conclusiones y reflexiones finales}

En el presente trabajo, de carácter exploratorio, se han presentado una serie de evidencias e hipótesis sobre las interrelaciones entre el grado de sincronización del ciclo del PIB total del Perú con los ciclos de los PIB de 31 países con los que el Perú comercia ${ }^{27}$, y el grado de integración comercial, financiera y de especialización sectorial del Perú con los mismos países. Además, se ha estimado la repercusión de los acuerdos preferenciales de comercio en estas interrelaciones. Sujeta a las limitaciones de las variables y mediciones y a la fragilidad estadística del método econométrico utilizado, la evidencia reportada en el presente trabajo revela dos conclusiones principales.

La primera es que existe una relación estadísticamente robusta entre el grado de sincronización de los ciclos de los PIB, la integración financiera y comercial y el grado de especialización sectorial. Sin embargo, las relaciones bidireccionales que tienen mayor robustez son aquellas entre la sincronización de los ciclos de los productos y el grado de integración financiera, entre esta y el grado de integración comercial, y de este último con la diferencia en el grado de especialización sectorial del Perú y sus países socios del comercio internacional. Una implicancia de estas interrelaciones es que incrementos en el tamaño relativo de los flujos comerciales del Perú

\footnotetext{
${ }^{27}$ Estos países explican más del $80 \%$ del flujo total de exportaciones e importaciones del Perú.
}

con respecto a los PIB reales de los 31 países socios comerciales podrían acrecentar el grado de sincronización de los ciclos de los PIB reales de aquellos países con el del Perú. Por otra parte, aunque con menor robustez estadística, aumentos en el diferencial del grado de especialización sectorial del Perú con respecto a sus países socios podrían también incrementar el nivel de sincronización de los ciclos de los PIB de dichos países La segunda conclusión que entrega la evidencia reportada es que, en general, los APC analizados no han incidido estadísticamente en la sincronización de los ciclos internacionales, la integración comercial y financiera y el grado de especialización sectorial.

Ambas conclusiones sugieren que el alto nivel de especialización sectorial de la economía peruana, particularmente de su sector transable ${ }^{28}$, y el mayor grado de integración comercial han ampliado la incidencia de los ciclos internacionales de los principales países socios comerciales en los ciclos del producto de la economía. Como consecuencia de ello, las políticas económicas dirigidas a diversificar los sectores productivos, los mercados de exportación, o ambos, pueden ayudar a atenuar, en el corto y largo plazo, los efectos negativos de las crisis internacionales.

\footnotetext{
${ }^{28}$ Donde cerca del $90 \%$ del valor exportado corresponde a bienes primarios intensivos en el uso de recursos mineros y agropecuarios.
} 
CUADRO A1

Indicadores de los grados de sincronización, integración comercial y financiera y especialización sectorial del Perú y sus principales

socios comerciales, 1982-2007

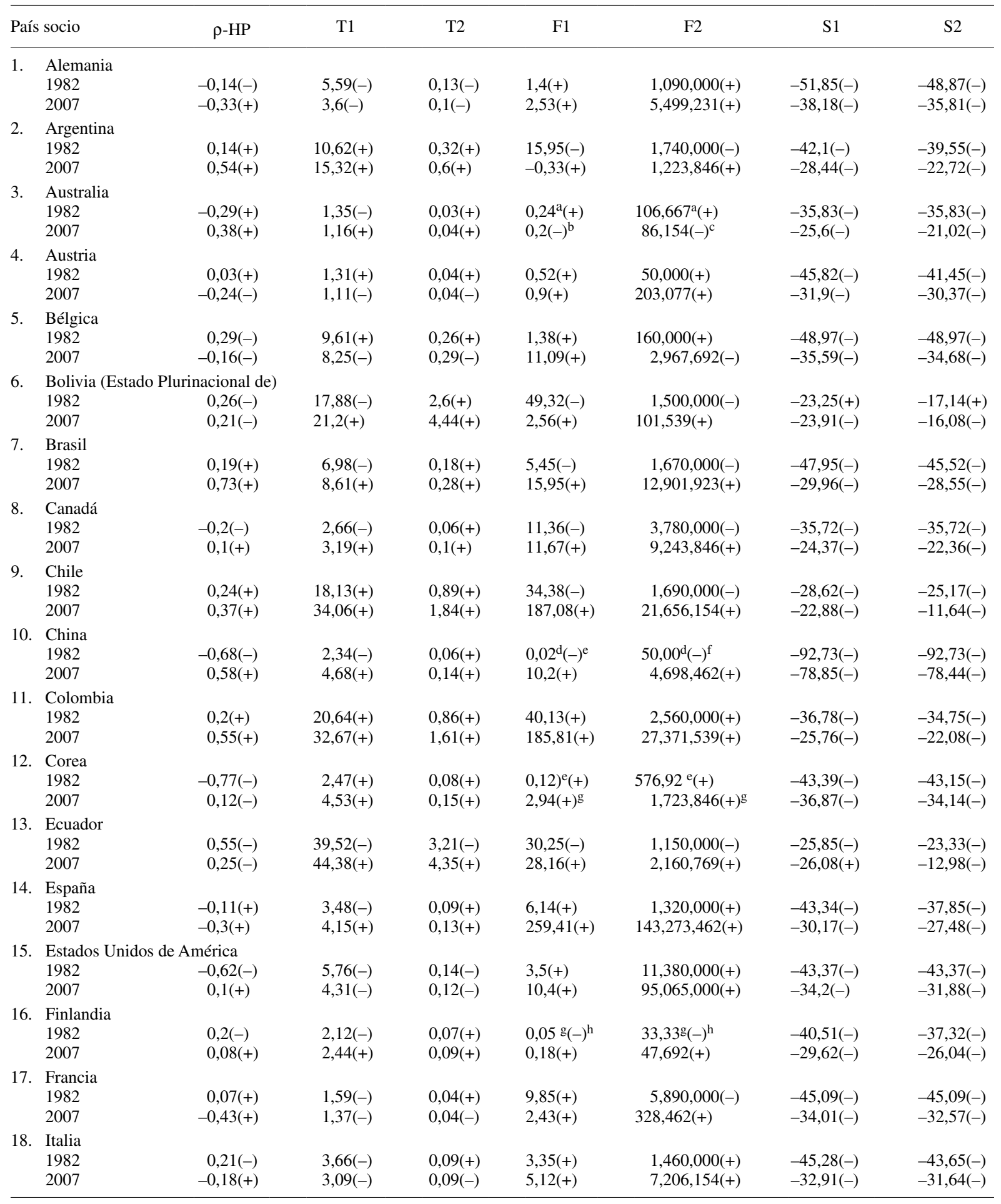


Continuación cuadro A1

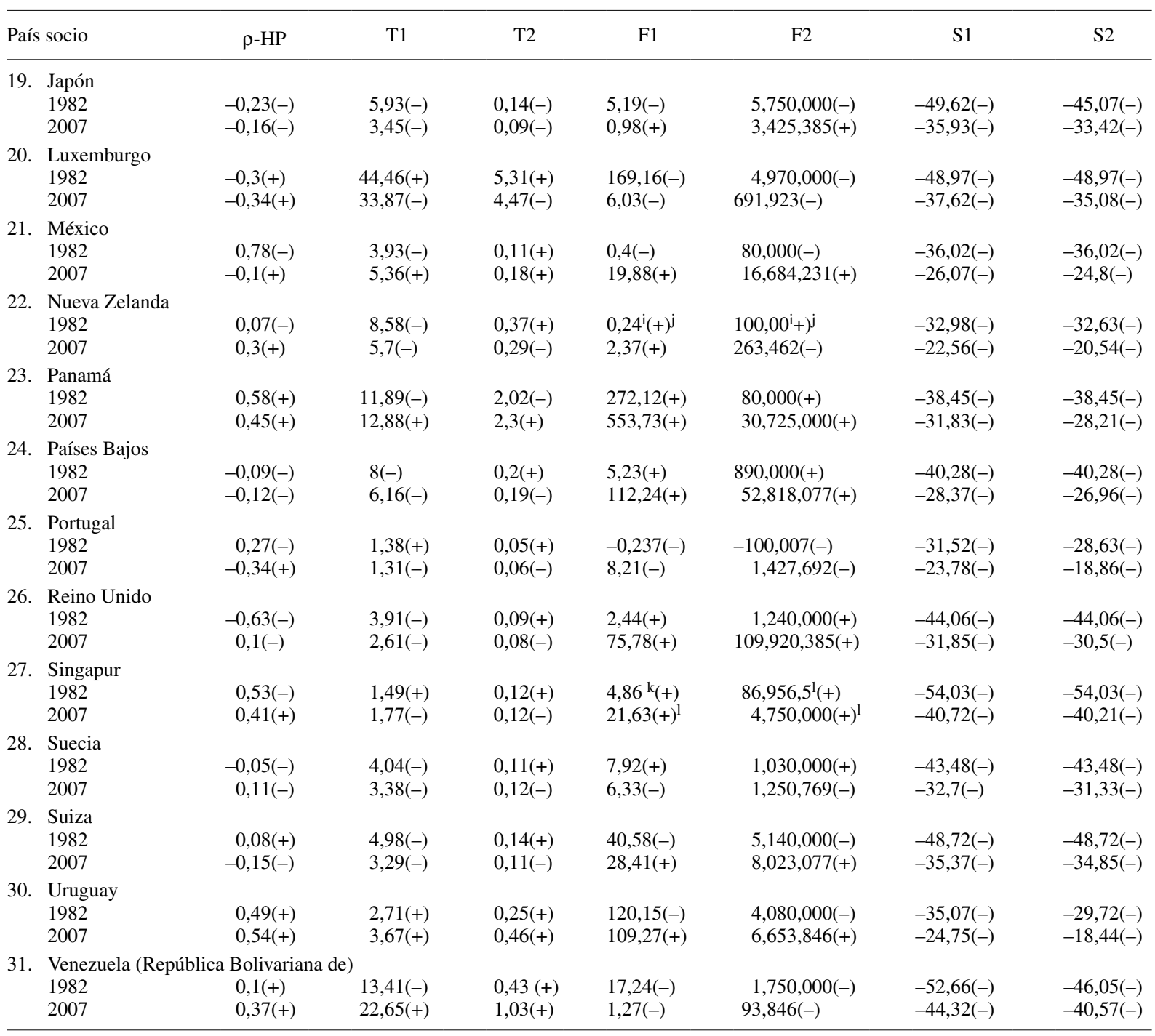

Fuente: elaboración del autor.

Notas: los indicadores $\rho$-HP, T1, T2, F1, F2, S1, S2 corresponden a los grados de sincronización de los ciclos, los dos de integración comercial, los dos de integración financiera, y los dos del grado de especialización definidos en la sección III.

Para los indicadores T1 y F1, las cifras están expresadas con E-04 y E-06 decimales respectivamente.

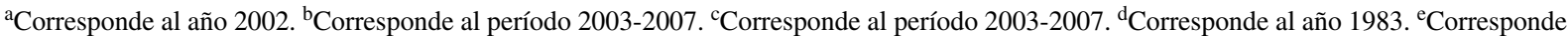

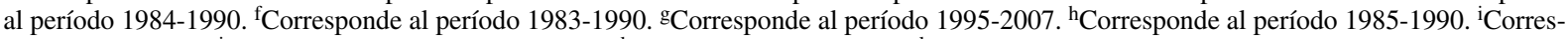
ponde al año 1985. jCorresponde al período 1986-1990. ${ }^{\mathrm{k}}$ Corresponde al año 2004. ${ }^{\mathrm{l} C o r r e s p o n d e}$ al período $2005-2007$. El indicador del grado de especialización, Si, está multiplicado por 100; el rango es de -200 a 0\%. Los signos entre paréntesis de los grados de sincronización corresponden al signo de las tasas de crecimiento de los coeficientes de correlación correspondientes al período 1982-1990 (para la fila del año 1982) y 1991-2006 (para la fila del año 2007). Así, un signo positivo en 1982 significa que el coeficiente de correlación se incrementó en el período 1982-1990. La información de SI es para el período 1982-2006. Los signos entre paréntesis para el resto de los indicadores corresponden a las tasas de variación anual de cada indicador en los períodos 1982-1990 y 1991-2007. 
Aghion, Ph. y A. Banerjee (2005), Volatility and Growth, Nueva York, Oxford University Press.

Ambler, S., E. Cardia y C. Zimmermann (2002), "International transmission of the business cycle in a multi-sector model", European Economic Review, vol. 46, № 2, Amsterdam, Elsevier.

Backus, D., P. Kehoe y F. Kidland (1993), "International business cycle: Theory and evidence", NBER Working Paper, N ${ }^{\circ}$ 4493, Cambridge, Massachusetts, National Bureau of Economic Research. Publicado también en Thomas Cooley (ed.), Frontiers of Business Cycle Research, Princeton, Princeton University Press, 1995.

(1992), "International real business cycles", Journal of Political Economy, vol. 100, № 4, Chicago, University of Chicago Press, agosto.

Baltagi, N. (2005), Econometric Analysis of Panel Data, West Sussex, John Wiley \& Sons.

Baxter, M. (1995), "International trade and business cycle", Handbook of International Economics, vol. 3, G. Grossman y K. Rogoff (eds.), Amsterdam, Elsevier.

Calderón, C., A. Chong y E.H. Stein (2007), “Trade intensity and business cycle synchronization: Are developing countries any different?", Journal of International Economics, vol. 71, № 1 , Amsterdam, Elsevier, marzo.

Calvo, G. y E. Mendoza (2000), "Rational contagion and the globalization of securities markets", Journal of International Economics, vol. 51, № 1, Amsterdam, Elsevier.

Canova, F. y H. Dellas (1993), "Trade interdependence and the international business cycle", Journal of International Economics, vol. 34, No 1-2, Amsterdam, Elsevier.

Coe, D.T. y E. Helpman (1995), "International R\&D spillovers", European Economic Review, vol. 39, № 5, Amsterdam, Elsevier.

Darvas, Z. y G. Vadas (2005), "A new method for combining detrending techniques with application to business cycle synchronization of the new EU members", MNB Working Papers, $N^{\circ} 2005 / 5$, Budapest, Magyard Nemzeti Bank.

De Gregorio, J. y J. Lee (2003), "Growth and adjustment in East Asia and Latin America", Documento de trabajo, $\mathrm{N}^{\circ} 245$, Santiago de Chile, Banco Central de Chile.

Edwards, S. (2007), "Crises and growth: A Latin American perspective", NBER Working Paper, N ${ }^{\mathrm{o}}$ 13019, Cambridge, Massachusetts, National Bureau of Economic Research.

Fidrmuc, J. e I. Korhonen (2009), "The impact of the global financial crisis on business cycles in Asian emerging economies", CESifo Working Paper, $\mathrm{N}^{\mathrm{o}} 2710$, Munich, CESifo Group Munich.

Frankel, J.A. y A.K. Rose (1998), "The endogeneity of the optimum currency area criteria", The Economic Journal, vol. 108, № 449, Royal Economic Society.

García-Herrero, A. y J. Ruiz (2008), "Do trade and financial linkages foster business cycle synchronization in a small economy?", Documento de trabajo, $\mathrm{N}^{\circ}$ 810, Madrid, Banco de España.

Heathcote, J. y F. Perri (2002a), "Financial autarky and international business cycles", Journal of Monetary Economics, vol. 49, $\mathrm{N}^{\circ} 3$, Amsterdam, Elsevier.

(2002b), "Financial globalization and real regionalization", NBER Working Paper, No 9292, Cambridge, Massachusetts, National Bureau of Economic Research.

Hodrick, R.J. y E.C. Prescott (1997), "Postwar US business cycles: An empirical investigation", Journal of Money, Credit, and Banking, vol. 29, № 1, Blackwell Publishing.

Imbs, J. (2004), "Trade, finance, specialization and synchronization", Review of Economics and Statistics, vol. 86, № 3, Cambridge, Massachusetts, The MIT Press.
INEI (Instituto Nacional de Estadística e Informática) (2009) [en línea] http://www.inei.gob.pe.

Kalemli-Ozcan, S., B. Sørensen y O. Yosha (2003), "Risk sharing and industrial specialization: Regional and international evidence", American Economic Review, vol. 93, N 3 , Nashville, Tennessee, American Economic Association, junio.

(2001), "Economic integration, industrial specialization, and the asymmetry of macroeconomic fluctuations", Journal of International Economics, vol. 55, $\mathrm{N}^{\mathrm{o}}$ 1, Amsterdam, Elsevier.

Kollman, R. (2001), "Explaining international comovements of output and asset returns: The role of money and nominal rigidities", Journal of Economics Dynamics and Control, vol. 25, № 10, Amsterdam, Elsevier.

Kose, M. y K. Yi (2006), "Can the standard international business cycle model explain the relation between trade and comovement?", Journal of International Economics, vol. 68, $\mathrm{N}^{\circ} 2$, Amsterdam, Elsevier.

(2002), "The trade comovement problem in international macroeconomics", Staff Reports, № 155, Nueva York, Banco de la Reserva Federal de Nueva York.

(2001), "International trade and business cycles: Is vertical specialization the missing link", American Economic Review, vol. 91, № 2, Nashville, Tennessee, American Economic Association.

Krugman, P. (1993), "Lesson of Massachusetts for EMU", The Transition to Economic and Monetary Union in Europe, F. Giavazzi y F. Torres (eds.), Nueva York, Cambridge University Press.

Lichtenberg, F. y B. van Pottelsberghe (1998), "International R\&D spillovers: A comment", European Economic Review, vol. 42, $\mathrm{N}^{\circ} 8$, Amsterdam, Elsevier.

Loayza, N. y V. Hnatkovska (2003), "Volatility and growth", Washington, D.C., Banco Mundial, inédito.

Lucas, R. (1990), “Why doesn't capital flow from rich to poor countries?", American Economic Review, vol. 80, N² 2, Nashville, Tennessee, American Economic Association, mayo.

Mendoza, E. (2002), "Credit, prices and crashes: business cycles with a sudden stop", Preventing Currency Crises in Emerging Markets, S. Edwards y J. Frankel (eds.), Chicago, University of Chicago Press.

Naciones Unidas (2009), "UN Comtrade” [en línea] http://comtrade. un.org/.

Stockman, A.C. (1988), "Sectoral and national aggregate disturbances to industrial output in seven European countries", Journal of Monetary Economics, vol. 21, № 2-3, Amsterdam, Elsevier.

Távara, J. y M.D. Tello (2010), "Productive development policies in Latin American countries: The case of Peru, 1990-2007", Documento de trabajo, $\mathrm{N}^{\circ} 129$, Washington, D.C., Banco Interamericano de Desarrollo.

Tello, M.D. (2011), "Barreras no arancelarias y protección interna y externa de los productos transables agropecuarios: El caso del Perú, 2000-2008", Política comercial, crisis externa e impactos sobre el sector agropecuario peruano, W. Mendoza y M.D. Tello (eds.), Lima, Consorcio de Investigación Económica y Social (CIES). (2009a), Arreglos preferenciales comerciales y crecimiento económico en América Latina, Lima, CENTRUm Católica.

(2009b), "Crecimiento económico, arreglos preferenciales comerciales y choques externos en el Perú, 1950-2007”, Crisis internacional: impactos y respuestas de política económica en el Perú, F. Jiménez y O. Dancourt (eds.), Lima, Pontificia Universidad Católica del Perú.

UNCTAD (Conferencia de las Naciones Unidas sobre Comercio y Desarrollo) (2009), UNCTAD Handbook of Statistics [http://stats.unctad.org/Handbook/TableViewer/tableView. aspx?ReportId=2061].

White, H. (1980), "A heteroskedasticity-consistent covariance matrix estimator and a direct test for heteroskedasticity", Econometrica, vol. 48, No 4, Nueva York, Econometric Society. 